\section{Investigation of a Constant Gradient Structure with Constant Iris Size}

\author{
Norbert Holtkamp ${ }^{1}$ \\ Stanford Linear Accelerator Center \\ Stanford University, Stanford, California 94309
}

\section{Abstract}

Travelling wave accelerators can be built either with constant impedance or constant gradient structures. Whereas for the constant impedance case the geometry of the cells does not change, the iris size in a constant gradient structure is decreased from cell to cell in order to slow down the power flow of the incoming $r f$ pulse by reducing the group velocity in order to achieve $P^{\prime}(z)=$ const. - Changing the phase advance per cell for the accelerating wave from the beginning towards the end of the structure provides as well a constant power loss per unit length and has the additional advantage of equal iris diameters over the entire length of the travelling wave tube. Concerning shor and long range wakefields, dark currents and mechanical demands, this type of constant gradient structure is discussed in comparison to the conventional one.

\section{Introduction}

Constant gradient (c.g.) structures are considered to be the essential components for the next generation linear collider main linacs. Apart from providing accelerating gradients of the order of $20-100 \mathrm{MeV}$ per meter. strong longitudinal and transverse wakefields as well as dark currents have to be

\footnotetext{
- Work supported by the Department of Energy, contract DE-AC03-76SF0051:
}

${ }^{1}$ visitor from the Technical University of Darmstadt, Germany
?

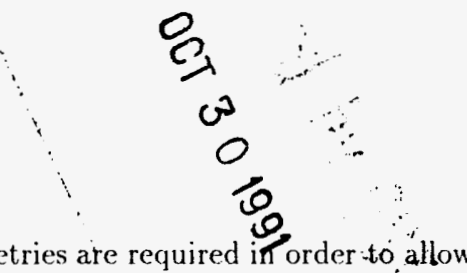

avoided. On the other hand simple geometries are required in order to allow mass production and efficient quality control to lower the structure costs.

In this paper a new type of c.g. structure is investigated, which keeps the iris diameter constant and therefore has a few advantages compared to the standard c.g. structure, where the iris diameter has to be changed by $20 \%$ to $30 \%$, depending on the overall design. Takeda et. al. [1] have pointed out that dark current can be amplified by a photomultiplier effect, because low energy electrons can easily hit the narrowing iris :s they travel down the structure. These electrons can extract a significant amount of the stored rf energy and may cause transverse emittance blowup due to the excited fields. This may limit the maximum gradient, that can be used for acceleration.

The short range wakefield for a given accelerating frequency is dominated by the iris size. The longitudinal wakes decrease roughly linearly and the transverse wakes quadratically with increasing hole diameters. The possibility of having a constant hole size is therefore especially of interest for linear colliders with higher bunch populations (single bunch colliders).

\section{Basic Features of a C. G. Structure}

Travelling wave tubes are normally characterized by a brillouin diagram (or dispersion diagram), which correlates the frequency of a wave with its wavelength in the structure. The phase velocity is given by: $c_{p h}=\omega / \beta$ where $\omega=2 \pi \cdot f_{0}$ with $f_{0}$ the accelerating frequency and $\beta=2 \pi / \lambda$ with $\lambda$ the wavelength. The group velocity, which is the velocity of power propagation in the tube, is given by the derivative: $v_{\mathrm{g}}=\partial \omega / \partial \beta$. As the electron velocity is approximately equal to the velocity of light $(=c)$, the crossing of the velocity of light line with the brillouin curve characterizes the wave in the tube which is synchronous with the particle.

In practice c.g. structures are normally built by changing the iris diameter along the structure. This affects essentially the bandwidth (or the cell to cell coupling) of the cells. It allows the adjustment the group velocity, because $\partial \omega / \partial \beta$ is changed for every wavelength except $\beta \cdot \mathrm{d}=0$ or $\pi$, where $\mathrm{v}_{\mathrm{g}}$ is equal to zero. An example for a brillouin curve is shown in figure 1 for a $11.424 \mathrm{GHz}$ c.g. structure, with $\mathrm{v}_{\mathrm{g}}=8 \%$ of $\mathrm{c}$ at the beginning and $3 \%$ of $\mathrm{c}$ at the end. 


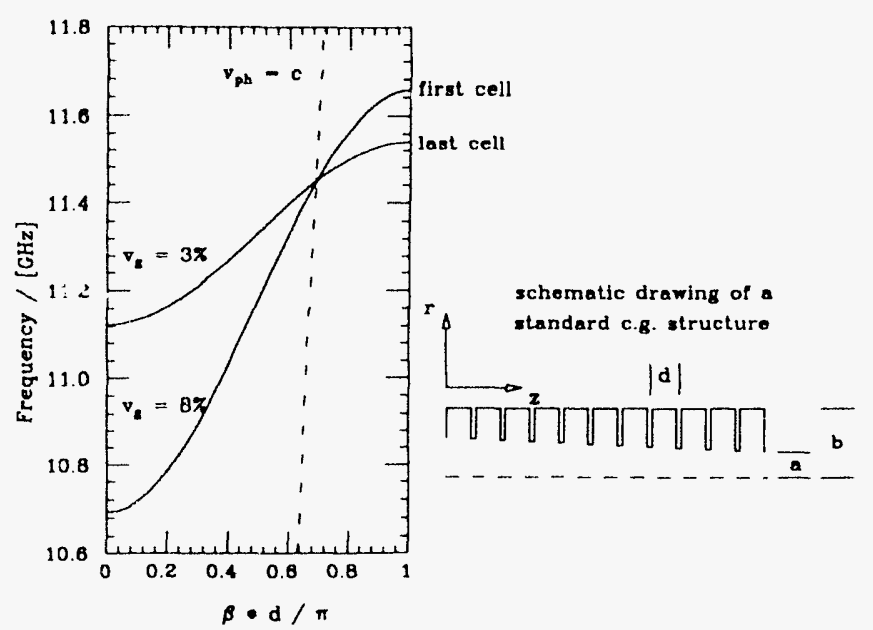

Figure 1: Brillouin diagram for a standard 11.424 (illz Structure The left picture shous the dispersion diagram for a 11.424 Gllz c.g. structure with $v_{g}=8 \%$ of $\mathrm{c}$ in the first cell and $\mathrm{v}_{\mathrm{g}}=3 \%$ of $\mathrm{c}$ in the last cell. $\mathrm{d}$ is the period (lingth) of the cells and $3 \cdot d$ gives the phase shift jer cell in mdians as the wave tmurels down the structure. This structure is operated in a $2 \pi / 3$ mode and therefore the relecity of light line must cross the brillouin curves at $\beta \cdot d / \pi=0.66$.

\section{Constant Gradient Structures with Con- stant Iris Diameter}

From one brillouin curve in figure 1, we can see another possibility to change $\partial_{w} / \partial ; 3$ at the crossing with the velocity of light line. Assuming the bandwidth to be constant.

$$
\Delta \mathrm{f}=[\mathrm{f}(\beta \cdot \mathrm{d}=\pi)-\mathrm{f}(\beta \cdot \mathrm{d}=0)]
$$

the crossing point can be shifted towards $\beta \cdot d=\pi$ in order to decrease the group velocity according to the decreasing slope of the curve. This results in a progressively increasing period (i.e. phase shift per cell) instead of reducing the iris diameter. Essentially the length of the gap is increased whereas the ir:s hole and the iris thickness keeps constant from the first to the last cell of the structure. A brillouin diagram and a schematic drawing for this type of travelling wave tubes is shown in figure 2 .

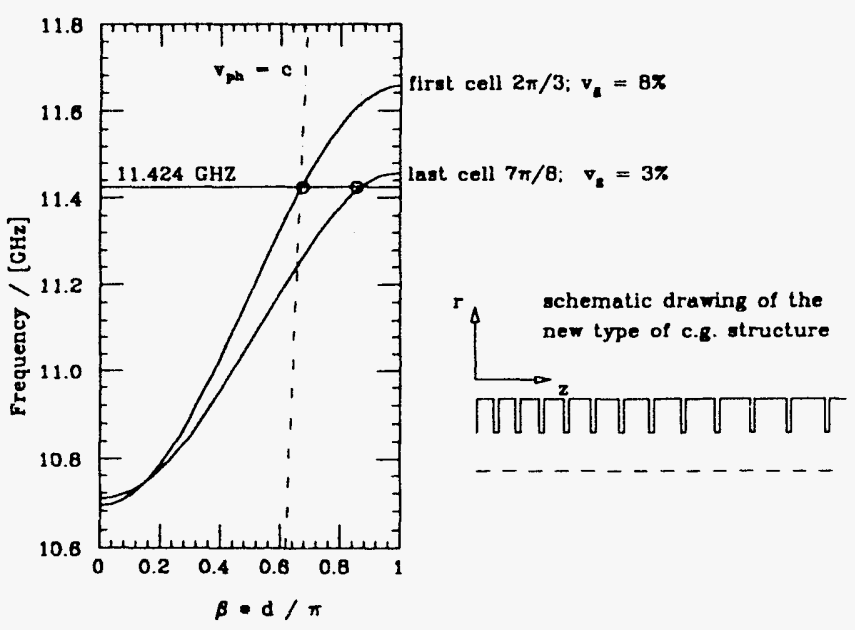

Figure 2: Brillouin diagram for a Structure with constant iris diameter Again the left picture shows the dispersion diagram for the first and the last cell of the c.g. structure. Due to the different phase shift per cell (different d). two velocity of light lines are drawn in the graph for the different cells. The group velocily again varies from $8 \%$ to $3 \%$ which is in this case equivalent to a change in phase shift from $2 \pi / 3$ to $i \pi / 8$. The schematic picture of the structure shows the increase of the gap length from the beginning to the end.

From the picture it is obvious too, that the bandwidth is not constant as the periodic length of the cells is increased, but the change is still significantly smaller for this type of structure.

\section{Comparison of Various Parameters}

In order to compare the advantages and disadvantages of both types of c.g. structure, a few parameters, which are normally of interest, have been 
calculated with various computer programs: KNTC [2]. TRANSVERS [3], IRMEL [1] and TBCI [5]

\section{The Geometry}

For both types of travelling wave tubes the parameters have been determined for the first and the last cell of a $11.424 \mathrm{GHz}$ structure. The geometry of the first cell was chusen to be the same for both types. The details are given in table 1 .

\begin{tabular}{|l|c|c||c|c|}
\hline & \multicolumn{2}{|c||}{ standard c.g. } & \multicolumn{2}{c|}{ new c.g. } \\
\hline & first cell & last cell & first cell & last cell \\
\hline $\mathrm{a} / \mathrm{cm}$ & 9.525 & 0.400 & 0.525 & 0.525 \\
$\mathrm{~b} / \mathrm{cm}$ & 1.1058 & 1.0500 & 1.1088 & 1.0977 \\
$\mathrm{~g} / \mathrm{cm}$ & 0.729 & 0.729 & 0.729 & 1.0024 \\
$\mathrm{~d} / \mathrm{cm}$ & 0.876 & 0.876 & 0.876 & 1.1484 \\
$\mathrm{r}$ & $2 \pi / 3$ & $2 \pi / 3$ & $2 \pi / 3$ & $7 \pi / 8$ \\
$\mathrm{r}_{\mathrm{g}}$ & $8 \%$ & $3 \%$ & $8 \%$ & $3 \%$ \\
\hline & \multicolumn{3}{|c}{} \\
\hline
\end{tabular}

Table 1: Geometry of the different cells

The table displays the different geometries uhich have been used to calculate various purameters for the two types of c.g. structure. The iris radius is given by a. the outer cavity radius by b. the gap by $\mathrm{g}$ and the periodicity by $\mathrm{d} . \mathrm{q}$ is the phase shift per cell (or the operating mode for the acclerating frequency). For the classical c.g. structure the geometry is similar to the one which is now under consideration for NLC at SLAC.

In both cases the group velocity roughly varies from $8 \%$ to $3 \%$. The iris radius or the gap length has been varied in order to achieve the required group velocity. the cavity radius $\mathrm{b}$ was used to tune the fundamental mode tc $11.24 \mathrm{Gilz}$ again and the disk thickness stayed constant. The reason for starting with a $2 \pi / 3$ mode for the constant iris c.g. structure will be explained later on (section: 5). The iris has square corners for all calculations.

\section{Results of the Calrulation}

Table 2 shows the result of various calculations. The longituoinal and transverse wake potential has been calculated for a $\sigma=1 \mathrm{~mm}$ long bunch and the maximum value within the bunch shape is presented in order to compare single bunch effects.

\begin{tabular}{|c|c|c|c|c|c|}
\hline parameter & units & \multicolumn{2}{|c|}{ standard c.g. } & \multicolumn{2}{|c|}{ new const. iris c.g. } \\
\hline & & first cell & last cell & first cell & last cell \\
\hline Shunt imp. R/Q & {$[\Omega / \mathrm{ml}]$} & 10600 & 14200 & 10600 & 8600 \\
\hline change & & \multicolumn{2}{|c|}{$+30 \%$} & \multicolumn{2}{|c|}{$-20 \%$} \\
\hline Max. $W_{\text {long }}$ & {$[\mathrm{V} / \mathrm{pC} / \mathrm{m}]$} & 464 & 520 & 464 & 435 \\
\hline change & & \multicolumn{2}{|c|}{$+12 \%$} & \multicolumn{2}{|c|}{$-6 \%$} \\
\hline $\operatorname{Max} . W_{\perp}$ & {$\left[\mathrm{V} / \mathrm{pC} / \mathrm{m}^{2}\right.$} & $0.8 \cdot 10^{5}$ & $1.2 \cdot 10^{5}$ & $0.8 \cdot 10^{5}$ & $0.5 \cdot 10^{5}$ \\
\hline change & & \multicolumn{2}{|c|}{$+40 \%$} & \multicolumn{2}{|c|}{$42 \%$} \\
\hline
\end{tabular}

Table 2: Various parameters for both types of c.g. structures

All values have bcen scaled to units per meter lingth of structure. The Q-value is in the range of $\mathrm{Q}=6900$ as calculated by URMIL. The change from the first to the last cell is given for every parameter in the line below. indicated by: change.

For the standard type of c.g. structure the shunt impedance rises towards the last. cell due to the smaller iris diameter. in contrast to the second type considered here. In the new type of structure the transit time factor decreases as the cell length is increased and therefore the shunt impedance drops towards the end.

\section{Power Consumption and Average Gradient}

Comparing the two geometries which have been calculated here, the average shunt impedance of the constant iris c.g. structure shown in table 2 is $25 \%(=30 \% / 2+20 \% / 2)$ less than the average shunt impedance of the standard c.g. structure. As the average gradient is proportional to:

$$
\overline{\mathrm{G}} \propto \sqrt{\mathrm{P}^{\prime} \cdot \mathrm{R}^{\prime}}
$$

$\mathrm{P}^{\prime}=$ peak power per unit length

$\mathrm{R}^{\prime}=$ shunt impedance per unit length 
this is equivalent to an increased power consumption of $25 \%$ for the constant iris geometry presented here, but the wake potentials are very different. For an exact comparison it may be more reasonable to tune the iris diameters of the new c.g. strcuture to have the same average shunt impedance, but this vas not done here. Average here is: mean value between the first and the last coll.

Nevertheless one can take the values of the last cell of the standard c.g. structure in table 2 and then scale these values according to the general tehavior of the constant iris c.g. structure. This estimate roughly shows, that for a constant iris c.g. structure with a reduced iris radius of $0.4 \mathrm{~cm}$. the average shunt impedance would be approximately equal ( $\approx 5 \%$ higher compared to the standard c.g. structure presented here. The scaling shows as well. that the average transverse wakefield would be the same (or a little tit smaller) in that case and the average longitudinal one wonld be slightly increased. The conclusion is. if power consumption and average gradient is the design goal for the accelerating structure. both types of travelling wave tubes are similar.

\section{Longitudinal and Transverse Wakefields: Single Bunch}

Due to the fact that most of the future linear colliders are designed for nultibunch operation or have extremely high single bunch charge in order to reach the maximum luminosity, transverse wakefields have to be kept as small as possible. Normally this is done by increasing the iris diameter, but this method approaches its natural limit. because the group velocity has to be controlled by the iris diameter as well. In the new type of constant iris c.g. structure the transverse wakefield is decreased by design because the iris diamcter stays constant within the structure. For the example given in table 2. the average transierse wakefield $W_{\perp}$ is decreased by $41 \%$ compared to the standard structure.

Additionally it is possible to handle a wider variety of group velccities dise to the fact. that once the group velocity is chosen for any mode in the fi:st cell. only the phase shift per cell has to be changed towards the end of the tube. but it has to be mentioned again that more power is required for this type of geometry (compare last section).

The variation of the longitudinal wakefield along the structure is only half as big for the constant iris c.g. tube. Comparing both types, this means as well that, designing for the same average shunt impedance, the beam loading is increased by roughly $3 \%$ (compare table 2 ) in the new type of c.g. tube.

For the geometries presented in table 1 and due to the fact, that the shunt impedance in the constant iris c.g. structure does not vary that much ( $20 \%$ instead of $30 \%$ ), the decrease of the longitudinal wakeficld is not as significant as for the transverse wakefield.

\section{Transverse Wakefields: Multi Bunch}

In order to avoid multibunch beam breakup. the transverse shunt impedance has to be as low as possible, especially for the first HEM mode which is normally the most severest one. In a standard c.g. structure part of this is included in the design, because the change in iris diameter affects most of the dipole modes very strongly. The situation can even be improved by using specific frequency distributions for the HEM mode cell to cell detuning within one structure, which may disturb the c.g. condition to a small amount but has the great advantage of a strongly lowered transverse wakefield. Thsi method was recently proposed at SLAC $[6,7]$, in order to improve the standard c.g. situation.

In the constant iris c.g. structure, the dipole mode frequency for a synchronous wave is essentially not affected, which is a great disadvantage compared to the standard type. The general behavior of the brillouin diagrams is presented in fig. 3 .

\section{Mechanical Differences and Difficulties}

In a classical c.g. structure with constant phase shift per cell (periodicity) the iris diameter is varied according to a certain change in group relocity, which is required to achieve a constant power loss per unit length. By changing the iris diameter the accelerating mode is only slightly affected and is tuned back by the cavity diameter. Therefore the hole size, its shape in the disk and the cavity radius have to be controlled to avoid phase errors. For example, in the NLC c.g. structure $27 \%$ change in iris radius is corrected by $5 \%$ change in cavity radius over the $1.5 \mathrm{~m}$ long structure to achieve a constant frequency for the $2 \pi / 3$ mode. After assembling the structure and due to the fact that the periodicity is constant, the tuning can be performed 

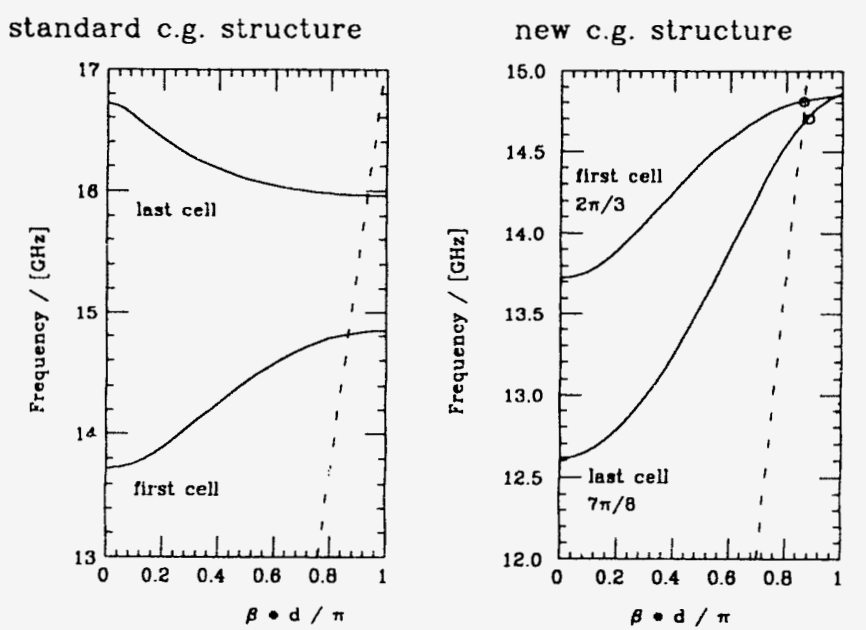

Figure 3: Brillouin diagram of the first HEM mode for both types of a $11.42 .1 \mathrm{GHz}$ structure.

The left picture shours the dispersion diagram of the first and the last cell in a stundard c.g. structure. Dus to the changc in iris diameter $(\approx 1.25 \mathrm{~mm})$, the dipole mode frequency of the first HEM has bcen shifted by approximately 1.1 GIIz or $7.5 \%$ near the point uhere the modes are synchronous with the particle. For the new type of structure, the first HEM mode is not shifted essentially in the synchronous region $(\approx 1 \%)$. as displayed in the right picture, which is a great disadvantage of this type of structure.

in a standardized way by moving a plunger from cell to cell.

For the constant iris c.g. structure, the periodicity changes by $27 \%$ whereas the cavity radius has to be corrected by only $1 \%$. The tuning is certainly more difficult, but various methods are possible to ach: ove the same accuracy $[8.9]$. The mechanical difficulties to provide quality control for a ch.nnging iris diameter and shape are assumed to be similar to changing the periodicity and kecping the varying phase advance within certain tolerances over a large number of cells.

\section{Design Constraints}

In order to construct a constant iris c.g. structure a few things are quite different compared to the standard type of structure and have to be considered. In order to go not too much into details, only a few approximations are presented.

\section{Calculation of the Group Velocity}

As mentioned before, the group velocity is given by the slope of the brillouin curve. For a travelling wave structure, which is used to accelerate velocity of light particles, the derivative at the intersection with the velocity of light line is therefore of special interest.

In order to calculate the group velocity for a certain wavelength, the slope of the brillouin curve has to be calculated at this point on the curve. The wavelength of a traveling wave in the iris loaded structure is given by:

$$
\lambda=\frac{2 \pi \cdot d}{\varphi}
$$

with: $\varphi=\beta \cdot d$, the phase shift per cell and $d=$ periodicity.

A commonly used approximation for the brillouin diagram is a cosine. describing the frequency as a function of $\varphi$ :

$$
\mathrm{f}(\varphi)=\frac{1}{2}\left(\mathrm{f}_{\max }+\mathrm{f}_{\min }\right)-\frac{1}{2} \Delta \mathrm{f} \cdot \cos (\varphi)
$$

( Compare $\Delta f$ to equation (1) and the curve to figure 1 or 2. )

From equation (4) the group velocity can easily be calculated to be:

$$
\begin{aligned}
\frac{\partial \omega}{\partial \beta} & =2 \pi \mathrm{d} \cdot \frac{\partial \mathrm{f}(\varphi)}{\partial \varphi} \\
\mathrm{v}_{\mathrm{g}} & =\pi \mathrm{d} \cdot \Delta \mathrm{f} \cdot \sin (\varphi)
\end{aligned}
$$

This implies, that for a fixed bandwidth and for fixed $d$ (=periodicity), the $\pi / 2$ mode has the maximum group velocity, which is certainly true if compared to figure 1 or 2 . 


\section{Group Velocity Considerations}

In a constant gradient tube, the group velocity has to be decreased from the first to the last cell. Therefore the first cell should start with a phase shift which provides the maximum group velocity.

The phase velocity of the wave has to be equal to the particle velocity in crder to be synchronous. Therefore the wavelength $\lambda$ in equation (3) must equal the free space wavelength:

$$
c=f_{0} \cdot \lambda
$$

with $f_{0}$ being the operating frequency. It follows from equation (3) that the periodicity $d$ can be expressed as a function of $\varphi$ with:

$$
\mathrm{d}=\frac{\lambda \cdot \varphi}{2 \pi}
$$

Dhe to the equations ( 7 ) and (6) the group velocity can be rewritten only dependent on $\varphi$ with:

$$
\frac{v_{g}(\varphi)}{c}=\frac{1}{2} \cdot \varphi \cdot \frac{\Delta f}{f_{0}} \cdot \sin (\varphi)
$$

and a plot of this curve is shown in fig. 4. For a fixed bandwidth the graph shows that the maximum group velocity is achieved for a phase advance of $\xi=116.24^{\circ}$. The exact value can be calculated by taking the derivative of equation (8) with respect to $\psi$ which gives an easy transcendental equation for $\varphi$ if set to zero:

$$
-\varphi=\tan (\varphi)
$$

This result is a bit surprising. due to the fact, that within a certain st ructure with fixed periodicity $d$ the maximum group velocity is always at $\tau=\pi / 2$. because the slope of the cosine has a maximum there. But in a travelling wave structure used for acceleration, the question is:

Which mode provides the maximum group velocity for a fixed bandwidth (or cell to cell coupling or iris size) of the structure? or to say it in other words:

How many disks per wavelength should be used in order to achieve the maximum group velocity?

and the answer is : approximately the $2 \pi / 3$ mode.

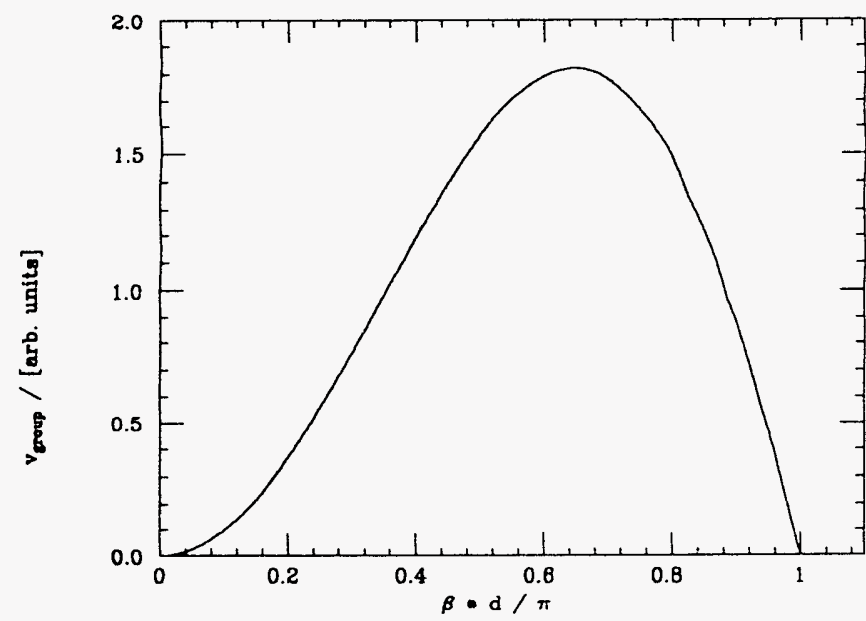

Figure 4: Group velocity as a function of phase advance per cell The group velocity as a function of phase advance per cell has a maximum at $\varphi=116.24^{\circ}$. For a fixed bandwidth, the group velocity similarly decreases on both sides of this value. As a matter of fact, the bandwidth is not constant and especially for the accelerating mode decreases with increasing $\varphi$. Calculations of the cavity pnrameters for a realistic constant iris c.g. structure show, that the group velocity decitases much faster for $\varphi \geq 2 \pi / 3$ and for large changes in $\varphi$ might even be dominated by the change in bandwidth.

Therefore all the calculations for the constant iris c.g. structure have been performed with a $2 \pi / 3$ mode st ructure for the first cell as mentioned in the section before. Apart from the fact that the bandwidth is not constant it should in principle be possible to construct a constant gradient structure gcing from $2 \pi / 3$ to $\pi / 3$, if the variation in group velocity which is required to have a constant gradient is not too large. 


\section{Summary}

In this paper, a new type of constant gradient structure with a constant iris hole size has be $n$ proposed and compared to a standard constant gradient structure. The performed calculations for a $11.424 \mathrm{GHz} \mathrm{X}$-band strurture have shown, that the required change in group velocity over the length of the travelling wave tube can easily be achieved by changing the phase shift of the accelerating mode per cell instead of decreasing the iris hole size. Concerning power requirements both types are similar.

Starting with the same iris diameter in the first cell, reduction of transverse and longitudinal wakefields is automatically performed but lowers the shunt impedance as well in the new type of structure. Nevertheless the overall goal might be achieving small wakefields than having high shunt impedance according to beam stability requirements. The amount of detuning of the dipole modes is shown to be much less than for convertional structures, which is the strongest argument against this type of travelling wave tubes.

Concerning dark currents and dark current enhancement due to photomultiplier effects, this structure has its strongest advantage because of the constant iris size if compared to the standard constant gradient structure. Mechanical as well as theoretical questions and comparis ons are considered but are not discussed in detail.

From the results listed above, one can conclude that the new type of constant gradient structure presented in this paper, does only solve some of the problems connected to the next linear collider main linacs, but nevertheless gives a new possibility of tuning and operating constant gradient structures.

\section{Acknowledgement}

I would like to thank R. Miller, P. Wilson and K. Bane for many interesting and time consuming discussions on all the subjects covered by this paper. I also want to thank for the support and interest of the whole Accelerator Theory group and of all the other poeple who helped me understanding the various problems connected to travelling wave accelerator structures. I also want to thank $K$. Bane and $R$. Miller for carefully reading this paper.

\section{References}

[1] S. Takeda. Proceedings of the Linear Collider Conference LC' 90, April 1990, KEK, Tsukuba, Japan.

[2] E. Keil, NIM, 100, (1972), pp. 419.

[3] K. Bane, B. Zotter, Proceedings of the $11^{\text {th }}$ Int. Conf. on High Energy Accelerators, CERN, (Birkhäuser Verlag, Basel, 1980), pp. 581.

[4] T. Weiland, On the Computation of Resonant Modes in Cylindrically Symmetric Cavities, NIM 216, (1983), p. 329-348.

[5] T. Weiland, Transverse beam cavity interaction part 1: short range forces, NIM 212, (1983), p. 13-21.

[6] J. W. Wang, Wakefield Measurements and Accelerator Structure Studies, SLAC, AAS Note 61, April, 1991.

[7] K. A. Thompson, J. W. Wang, Simulation of Accelerating Structures with Large Staggered Tuning, SLAC, IEEE Particle Accelerator Conference. May 6-9, 1991, San Francisco, to be published.

[8] H. Hoag, H. Deruyter, private communications

[9] R. Miller, private communications

\section{DISCLAIMER}

This report was prepared as an account of work sponsored by an agency of the United States Government. Neither the United States Government nor any agency thereof, nor an; of their employees, makes any warranty, express or implied, or assumes any legal liability or responsibility for the accuracy, completeness, or usefulness of any information, apparatus, product, or process disclosed, or represents that its use would not infringe privately owned rights. Reference herein to any specific commercial prociuct, process, or service by trade name, trademark, manufacturer, or otherwise does not necessarily constitute or imply its endorsement, recommanufacturer, or otherwise does not necessarily constitute or imply its endorsement, recom-
mendation, or favoring by the United States Government or any agency thereof. The views and opinions of authors expressed herein do not necessarily state or reflect those of the United States Government or any agency thereof. 

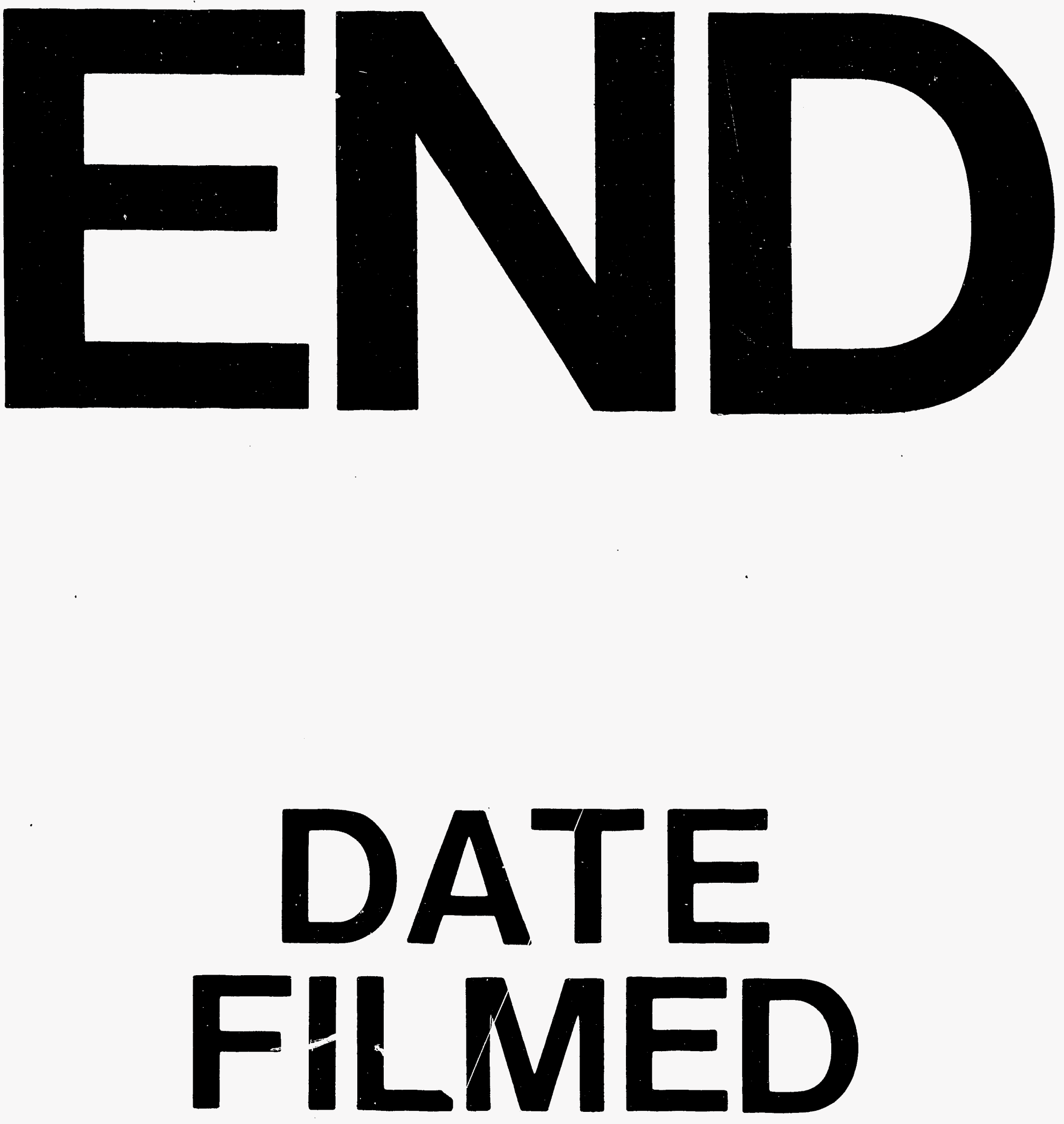

1

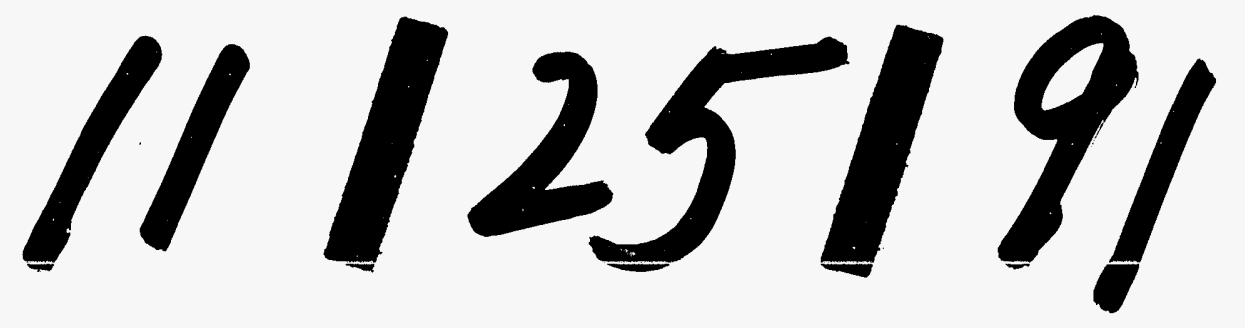

$\overline{1}$ 
